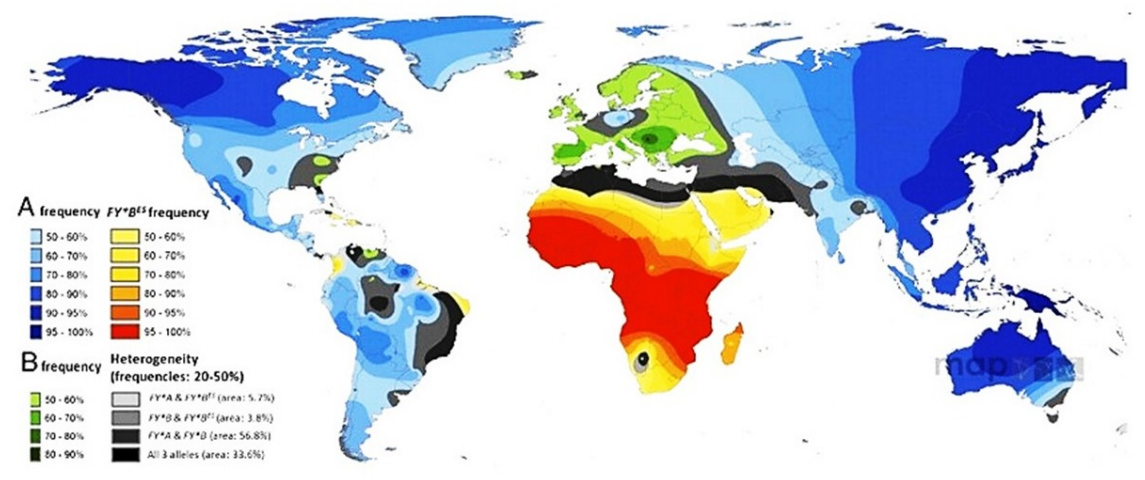

\title{
African or African American? That is the question. The Duffy antigen and the Chemokine Storm in Covid-19
}

\author{
Carlos Rodriguez \\ 1 Fundación Jiménez Díaz \\ Funding: The author(s) received no specific funding for this work. \\ Potential competing interests: The author(s) declared that no potential competing interests exist.
}

\section{Abstract}

African Americans have been heavily hit by the COVID-19 pandemic. The reasons for the specially severe forms of the disease in Black Americans have not been fully uncovered. I present here a hypothesis that arguably gives the blood group marker and chemokine decoy receptor Duffy protein a central role in quenching the Chemokine Storm as present in the severe forms of the COVID-19 disease.

Among the many facts that the pandemic had brought to the surface, one has touched me especially: Black people in America (North and South alike) are massively overrepresented in the coronavirus death statistics ${ }^{[1]}$. In sharp contrast to this, Black people living in Africa seem to be "protected" from the virus (and this is not an overstatement: just look at the statistics!). This protection is not due to age issues (it has been argued ${ }^{[2]}$ without scientific substance that Africans suffer a milder version of Covid- 
19 because they are younger. Younger than whom? As I said, no sound science in that argument) nor to climate factors. So, what are the real reasons for this apparent paradox? Let's try to expand here on a couple of arguments that I think solidly withstand the scientific challenge.

Something that the medical literature has been researching for many years is the fact that African people, after millenia of cohabitation with parasitic diseases, have been naturally selected with gene alleles that make them "less visible" to lethal plagues like, for instance, malaria. Since the early ages, malaria has spread death in the hundreds of millions throughout Africa. The way nature has chosen to get around the endemicity of such a deadly disease has been through the slow selection of genes that make people less prone to being infected or else to suffer the fatal complications of the disease. These genetic mutations are themselves the cause of diseases but, in the malaria historical context, create a population balance tilted towards more survival. Conditions that are present in both Africans from Africa and Africans from the African Diaspora, include several forms of anemia like sickle cell disease ${ }^{[3]}$, Thalasemia ${ }^{[4]}$, or the so-called G6PD deficiency ${ }^{[5]}$ among many others ${ }^{[6]}$. Many of these mutations affect genes that code for erythrocyte proteins (not surprising, since the malaria parasite spends most of its human life cycle within blood erythrocytes). Below is a summary of relevant erythrocyte gene variants linked to malaria protection (borrowed from Kwiatkowski DP. ${ }^{[7]) .}$

\begin{tabular}{|c|c|c|c|}
\hline Gene & Protein & Function & Reported Genetic Associations with Malaria \\
\hline$F Y$ & Duffy antigen & Chemokine receptor & $\mathrm{FY}^{*} \mathrm{O}$ allele completely protects against $P$. vicax infection. \\
\hline$G 6 P D$ & $\begin{array}{l}\text { Glucose-6-phosphatase } \\
\text { dehydogenase }\end{array}$ & Enzyme that protects against oxidative stress & G6PD deficiency protects against serere malaria. \\
\hline GYPA & Glycophorin A & Sialoglycoprotein & GYPA-deficient erythrocytes are resistant to invasion by $P$. faliciparum. \\
\hline GYPB & Glycophorin B & Sialoglycoprotein & GYPB-deficient erythrocytes are resistant to invasion by $P$. falciparum. \\
\hline GYPC & Glycophorin C & Sialoglycoprotein & GYPC-deficient erythrocytes are resistant to invasion by $P$. falciparum. \\
\hline$H B A$ & $\alpha$-Globin & Component of hemoglobin & $\begin{array}{l}\alpha^{+} \text {Thalassemia protects against severe malaria but appears to enhance mild } \\
\text { malaria episodes in some environments. }\end{array}$ \\
\hline$H B B$ & $\beta$-Globin & Component of hemoglobin & $\begin{array}{l}\mathrm{HbS} \text { and } \mathrm{HbC} \text { alleles protect against serere malaria. HbE allele reduces parasite } \\
\text { invasion. }\end{array}$ \\
\hline HP & Haptoglobin & $\begin{array}{l}\text { Hemoglobin-binding protein present in } \\
\text { plasma (not erythrocyte) }\end{array}$ & $\begin{array}{l}\text { Haptoglobin 1-1 genotype is associated with susceptibility to serere malaria in } \\
\text { Sudan and Ghana. }\end{array}$ \\
\hline SCL4AI & $\begin{array}{l}\text { CD233, erythrocyte band } \\
3 \text { protein }\end{array}$ & Chloride bicarbonate exchanger & Deletion causes ovalocytosis but protects against cerebral malaria. \\
\hline
\end{tabular}

Figure 1

On the top of the list you will appreciate the mention of a protein called Duffy. 
The Duffy antigen is in itself important enough, for its expression on the surface of erythrocytes alone defines a blood group system. Indeed, polymorphisms in this gene are the basis of the Duffy Blood Group System ${ }^{[8]}$. Early on, it was found that the overwhelming majority of people of African descent had the erythrocyte phenotype Fy(ab-) (henceforth Duffy (-)) :68\% in African Americans and 88-100\% in African people (including more than $90 \%$ of West African people). In Black People, this phenotype silences the gene in erythrocytes (something like the O group, that is defined by the absence of A and B proteins). By the way, this Duffy (-) phenotype is exceedingly rare in White People/Caucasians ${ }^{[9]}$. Because the Duffy antigen is uncommon in those of African descent, the presence of this antigen has been used to detect genetic admixture. In a sample of unrelated African Americans ( $n=235)$, Afro-Caribbeans ( $n=90)$ and Colombians ( $n=93$ ), the frequency of the $-46 T$ (Duffy positive) allele was $21.7 \%, 12.2 \%$ and $74.7 \%$ respectively ${ }^{[10]}$. As a short recap of all of the above, most Black Africans and a majority of Out of Africa Black People (OAB) are Duffy (-) (i.e. they don't have the Duffy protein on their red blood cells).

On top of the list in figure 1, you will also see mentioned that the Duffy (-) phenotype protects people against several strains of the Plasmodium parasite (the cause of malaria). Although somewhat controversial, the reason for the evolutionary selection of a Duffy (-) phenotype in African descent is, at least partly, because the Duffy protein acts as a receptor for the parasite on the erythrocyte surface. Hence, erythrocytes with no Duffy on their cell membrane will be resistant to the infection by the Plasmodium parasite. Or so the story goes. But to delve into this will only distract us from our main focus, which is the third mention on the first row of the list above:

The Duffy antigen is also a broad receptor of chemokines in human blood. But "why would this be so relevant to the Covid-19 story?" You may ask.

So far, in the Covid-19 abundant literature you have heard a lot about "Cytokine Storms" but nothing in the way of "Chemokine Storms", right?

I hadn't either. Not until the other day.

On that day, my colleague sent me a paper over Whatsapp ("One of those thousands of papers from the paper tide that I have to read!", I thought). The paper was intriguingly entitled "Is a "Cytokine Storm" Relevant to COVID-19?"[11]. In a nutshell, the authors rejected the value of the "cytokine storm" notion as pathog enesis coin for the disease. 
Instead, they argued that the cytokine storm is not such (at least in most cases of ARDS in Covid-19) and suggested to look somewhere else to explain the undoubtedly positive effects of drugs like the corticosteroid Dexamethasone in the disease.

So, the next day, when my colleague asked me about the paper, I, as usual, prompted a quick impulsive answer: "I don't know", I said, "maybe it is not a Cytokine Storm but a Chemokine Storm". This came out of nowhere, but, at the same time, straight from my mouth. "What?" Asked my colleague in disbelief. "I don't know. I only know that chemokines are the traffic lights that decide almost everything during an inflammatory response. And, importantly, many of them are really through the roof in this disease, both in the blood ${ }^{[12][13][14]}$ and in the lungs $s^{[15]}$, and, in addition, chemokine receptor inhibition has already been proposed as a potential treatment for the disease ${ }^{[16] "}$. I said something like this, leaving my colleague plunged into deep thoughts,.

This short exchange however, as usual, sparked my curiosity and I started reading (more) about chemokines. Among those readings, an excellent review (and a very old one, by today's standards) caught my attention ${ }^{[17]}$. Reading the paper, I could recall what I already knew: that chemokines are small peptides that activate so-called GPRs, seven transmembrane protein receptors on the surface of cells (mosty on inflammatory cells like neutrophils, macrophages, lymphocytes, eosinophiles, etc.). I also knew already that levels of chemokines in blood and tissues create molecular gradients that serve as cues for those cells to enter inflamed tissues or to egress from them. But I also learnt new important things that I summarize here:

1. Chemokines can have inflammatory or homeostatic functions or both

2. Chemokines are activators of their putative receptors but they can also be antagonists of other chemokines by the non-productive binding to the receptors of the latter chemokines.

3. Most relevant to this post, I also learnt of the existence of so-called chemokine DECOY RECEPTORS.

And, as thoroughly explained in the review, the Duffy protein was apparently one of those decoy receptors, in fact, the most important one.

But what is this protein anyway? The Duffy protein (which, by the way, is named after the patient in which it was discovered) also known as Fy glycoprotein (FY) or CD234 (Cluster of Differentiation 234), is a protein that in humans is encoded by the DARC gene. It codes 
for a seven transmembrane domain cell surface protein mainly expressed on human erythrocytes but also on other cells from liver, spleen, kidney and brain ${ }^{[18]}$.

As mentioned before, besides being a receptor for some Plasmodium parasites, the protein has a very important role in inflammation and tissue homeostasis. And this is because it serves as a (decoy) receptor of some if not all of the $40+$ known chemokines.

So, let's take a little back view at how the story began. It was back in nineties in the Bay Area, where a company named Genentech had just discovered the first chemokines and was looking to develop antibodies against one that, at the time, was already suspected to be involved in, you guessed it, major inflammatory processes: the CXCL8, also known as IL-8 chemokine. One of the questions that researchers asked was if, as happens with many other hormones, there was a major IL-8 binding protein in the human plasma. To their surprise, what they found instead was that erythrocytes bound IL-8 in a high affinity $(5 \mathrm{nM})$ and saturable manner ${ }^{[19]}$. Interestingly, it was not always possible to successfully detect CXCL8 binding to blood samples and it appeared that the blood from these CXCL8 non-responders was always from African American donors ${ }^{[20]}$.

Since then, we have learned many things about the Duffy protein as a broad chemokine receptor. It is apparently able to bind with high affinity to most chemokines of the CC and CXC types (the majority of chemokines are of these two classes) ${ }^{[21]}$. The Duffy protein, unlike most seven transmembrane receptors, is not coupled to a G-protein or any other intracellular signalling system; thus it is incapable of generating second messages inside the cell, something that we could infer from its host cell, the erytrocyte, a hemoglobin packed carcase with no nucleus ${ }^{[22]}$. Hence, the term decoy (or sink) receptor: a receptor that does not send messages inside the cell but that uniquely functions to take chemokines away from the circulation.

A variety of research has shown that the role of the Duffy protein could be as a broad regulator of numerous inflammatory processes like atherosclerosis ${ }^{[23]}$, respiratory viral infections $^{[24]}$, or Inflammatory Bowel Disease ${ }^{[25]}$.

But perhaps the most telling research favoring a key role of the Duffy antigen in inflammation comes from the work in mice. Indeed, and fortunately, mice express a Duffy homologous on murine erythrocytes and Duffy knockouts (Fy-/- mice) have been extensively studied. Homozygous deletions in mouse genes are behind many major advances in understanding the function of a huge number of human genes. Particularly, 
knowledge in human immunology would be very far from what it is today were not for the pioneering studies in mouse knockouts. Early studies on the homozygous deletion of the Duffy gene in mice were somehow disappointing, since no particular abnormal phenotype was found in those animals, leading the authors to conclude then that the Duffy protein is somewhat functionally redundant in mice ${ }^{[26]}$. However work (then and later on) on challenged Fy-/- mice have brought unequivocal results: In mice homozygous for the deletion, there was less neutrophil recruitment into the peritoneal cavity and neutrophil influx in the intestines and lungs than in wild-type mice ${ }^{[26]}$. Mice lacking the Duffy receptor present an increased lung inflammation after LPS challeng $\mathrm{e}^{[27]}$ . Importantly for the COVID-19 argument, deletion of the Duffy antigen induces glucose intolerance and insulin resistance independent of body weight or adiposity ${ }^{[28]}$.

To summarize, I really believe that black people living for generations in the New Continent have kept gene alleles that, besides not being anymore protective against parasite diseases that are almost absent in most of the American Continent, in addition, can represent a heavy ballast when facing the worst complications of the Covid-19 disease. In particular, the absence of the so-called Duffy gene in African American people could be one of the factors (if not the factor) that explains the high mortality rate of Covid-19 among black people in the Americas.

Now to the bright side of the story: What about Black Africans? After all, they are an almost 100\% Duffy (-) population. We know too well that genetic polymorphisms are either promoted or deleted in populations over very extended periods of time. This is what happened in Africa over the centuries. African people had to deal with malaria for millenia, so their genes have made twitches and adaptations accordingly, mainly aimed at avoiding the disease or its complications.

So far, we can only explain the high prevalence of the Duffy (-) allele in Africans. But what about the clearly milder COVID-19 disease in Sub-Saharan Africa? After all, throughout the paper, we have argued just the opposite, that people with the Duffy (-) phenotype are high risk gropu for the Chemokine Storm, right? So, how can we reconcile this apparent contradiction. Well, this is when the other aspect of the historical cohabitation of humans and parasites (and my favorite story line) comes into play. The true main characters in this sequel, you figured it out, are helminths. Parasitic worms have been around humans in the African continent for ages and, as we have previously postulated ${ }^{[29]}$, they have shaped people's immune system in such a way that now, this alone, represents a real evolutive advantage when facing the Covid-19 pandemic. Simply explained: most Sub- 
Saharan Africans, when infected by COVID-19, and because they have a helminth shaped Th2 like immune system, they simply won't develop any Chemokine Storm whatsoever. Because of that, they won't need the Duffy protein, since there isn't any Chemokine tide to counter. Does it make sense to you?

As a conclusion then, the helminth driven Th2 tilted immune system in African populations would explain why African Blacks, despite having Duffy-less erythrocytes, unlike their American relatives, are mostly protected against COVID-19.

To end up with another positive note, here are a few suggestions for those who have access to research facilities or to related investig ative means:

- A straightforward approach, and one we can directly think of from this paper, would be to use Duffy (+) Red Blood concentrates in Duffy (-) people with a Chemokine Storms like the one in the Acute Respiratory Distress Syndrome of COVID-19. In the same vein, another related approach is the use of reconstituted lisosomes. Indeed, fully functional seven transmembrane receptors can be successfully reconstituted in liposomes ${ }^{[30]}$, so it would be interesting to test the therapeutic activity of functional Duffy receptor liposomes to counter the putative Chemokine Storm in Covid-19. This approach would circumvent possible histocompatibility/contamination issues that would be associated with the use of hemoconcentrates.

- It has been shown that the $\mathrm{N}$-terminal domain of chemokine receptors is the one that makes contact with chemokines. This raises the question of the feasibility of producing and isolating a druggable soluble Duffy $\mathrm{N}$-terminal domain that can be used in animal models of Covid-19 disease ${ }^{[31]}$ and eventually as a drug to counter or abolish the development of SARS.

- We could also ask the following questions: What are the Duffy blood groups in White People at large and in people with Covid-19 and, most importantly, in White People with severe complications of the disease? By the same token, is there a correlation between Duffy (-) homozygosity or Duffy (+/-) heterozygosity in NonBlack patients and disease severity? This kind of studies would provide us with a broad picture of the real role of the Duffy gene and, by extension, of the Chemokine Storm in the pathophysiology of the COVID-19 disease.

\section{References}


1. ^ C. D. Turner. (2020). Covid-19 The Extinction Of The Black Race. corey turner.

2. ^Issideen Ayinla Osseni. (2020). COVID-19 pandemic in sub-Saharan Africa: preparedness, response, and hidden potentials. Trop Med Health, vol. 48 (1). doi:10.1186/s41182-020-00240-9.

3. ^Vernon M. Ingram. (2004). Sickle-Cell Anemia Hemoglobin: The Molecular Biology of the First "Molecular Disease"-The Crucial Importance of Serendipity. Genetics, vol. 167 (1), 1-7. doi:10.1534/genetics.167.1.1.

4. ^ V. M. INGRAM, A. O. W. STRETTON. (1959). Genetic Basis of the Thalassæmia Diseases. Nature, vol. 184 (4703), 1903-1909. doi:10.1038/1841903aO.

5. ^ P. E. CARSON, C. L. FLANAGAN, C. E. ICKES, A. S. ALVING. (1956). Enzymatic Deficiency in Primaquine-Sensitive Erythrocytes. Science, vol. 124 (3220), 484-485. doi:10.1126/science.124.3220.484-a.

6. ^ P. Ligoxygakis. (2001). Malaria resistance genes in humans. Trends in Genetics, vol. 17 (9), 494. doi:10.1016/s0168-9525(01)02460-x.

7. ^ Dominic P. Kwiatkowski. (2005). How Malaria Has Affected the Human Genome and What Human Genetics Can Teach Us about Malaria. The American Journal of Human Genetics, vol. 77 (2), 171-192. doi:10.1086/432519.

8. ^ Christof Weinstock, Joannis Mytilineos, Peter Bugert, Nicole Sitzmann, Elke Pensel, Hubert Schrezenmeier. (2019). A novel allele of the atypical chemokine receptor 1 (ACKR1) gene containing the nucleotide change C.126 T>G (p.42G/u) encodes a third Duffy blood group protein sequence antithetical to that encoding Fyaand Fybantigens. Transfusion, vol. 59 (6), 2158-2159. doi:10.1111/trf.15232.

9. ^ Marion E. Reid, Christine Lomas-Francis. (2004). Duffy blood group system. doi:10.1016/b978-012586585-2/50011-1.

10. ^ Renate G Nickel, Stephanie Ann Willadsen, Linda R Freidhoff, Shau-Ku Huang, Luis Caraballo, Raana P Naidu. (1999). Determination of Duffy genotypes in three populations of African descent using PCR and sequence-specific oligonucleotides. Human Immunology, vol. 60 (8), 738-742. doi:10.1016/s0198-8859(99)00039-7.

11. ^ Pratik Sinha, Michael A. Matthay, Carolyn S. Calfee. (2020). Is a "Cytokine Storm" Relevant to COVID-19?.JAMA Intern Med. doi:10.1001/jamainternmed.2020.3313.

12. ` Ying Chi, Yiyue Ge, Bin Wu, Wenshuai Zhang, Tao Wu, Tian Wen. (2020). Serum Cytokine and Chemokine Profile in Relation to the Severity of Coronavirus Disease 2019 in China. doi:10.1093/infdis/jiaa363.

13. ^ Joseph Balnis, Alejandro P Adam, Amit Chopra, Hau C Chieng, PaulJ Feustel, Katherine A Overmyer. (2020). Higher plasma levels of Chemokine CCL19 are associated with poor SARS-CoV-2 acute respiratory distress syndrome (ARDS) outcomes.. doi:10.1101/2020.05.21.20051300. 
14. ^ Francesca Coperchini, Luca Chiovato, Laura Croce, Flavia Magri, Mario Rotondi. (2020). The cytokine storm in COVID-19: An overview of the involvement of the chemokine/chemokine-receptor system. Cytokine \& Growth Factor Reviews, vol. 53, 25-32. doi:10.1016/j.cytogfr.2020.05.003.

15. ^ Robert Lorenz Chua, Soeren Lukassen, Saskia Trump, Bianca P. Hennig, Daniel Wendisch, Fabian Pott. (2020). COVID-19 severity correlates with airway epitheliumimmune cell interactions identified by single-cell analysis. Nat Biotechnol. doi:10.1038/s41587-020-0602-4.

16. ^ Shiyu Li, Yuzhe Wang, Lixia Feng, Zhenyou Jiang, Feng Cong, Youyu Chen. (2020). Chemokine Receptor Inhibitor VMIP-II Promoting Lymphocytes in COVID-19 Patients and Its Related Mechanism In Vitro. doi:10.21203/rs.3.rs-33165/v1.

17. ^Clemens Esche, Cristiana Stellato, Lisa A. Beck. (2005). Chemokines: Key Players in Innate and Adaptive Immunity. Journal of Investigative Dermatology, vol. 125 (4), 615628. doi:10.1111/j.0022-202x.2005.23841.x.

18. ^ A. Chaudhuri, J. Polyakova, V. Zbrzezna, K. Williams, S. Gulati, A. O. Pogo. (1993). Cloning of glycoprotein D CDNA, which encodes the major subunit of the Duffy blood group system and the receptor for the Plasmodium vivax malaria parasite.. Proceedings of the National Academy of Sciences, vol. 90 (22), 10793-10797. doi:10.1073/pnas.90.22.10793.

19. ^ W C Darbonne, GC Rice, M A Mohler, T Apple, CA Hébert, A J Valente. (1991). Red blood cells are a sink for interleukin 8, a leukocyte chemotaxin.. J. Clin. Invest., vol. 88 (4), 1362-1369. doi:10.1172/jci115442.

20. ^ Richard Horuk. (2015). The Duffy Antigen Receptor for Chemokines DARC/ACKR1. Front. Immunol., vol. 6. doi:10.3389/fimmu.2015.00279.

21. ^ Lu Zhao-hai, Wang Zi-xuan, Richard Horuk, Joe Hesselgesser, Lou Yan-chun, Terrence J. Hadley. (1995). The Promiscuous Chemokine Binding Profile of the Duffy Antigen/Receptor for Chemokines Is Primarily Localized to Sequences in the Aminoterminal Domain.J. Biol. Chem., vol. 270 (44), 26239-26245. doi:10.1074/jbc.270.44.26239.

22. `Stavros Apostolakis, Georgios K Chalikias, Dimitrios N Tziakas, Stavros Konstantinides. (2011). Erythrocyte Duffy antigen receptor for chemokines (DARC): diagnostic and therapeutic implications in atherosclerotic cardiovascular disease. Acta PharmacolSin, vol. 32 (4), 417-424. doi:10.1038/aps.2011.13.

23. `Stavros Apostolakis, Demetrios Spandidos. (2013). Chemokines and atherosclerosis: focus on the CX3CL1/CX3CR1 pathway. Acta Pharmacol Sin, vol. 34 (10), 1251-1256. doi:10.1038/aps.2013.92.

24. ^ Virginia Amanatidou, Apostolos Zaravinos, Stavros Apostolakis, Demetrios A. 
Spandidos. (2011). Chemokines in Respiratory Viral Infections: Focus on Their

Diagnostic and Therapeutic Potential. Crit Rev Immunol, vol. 31 (4), 341-356.

doi:10.1615/critrevimmunol.v31.i4.40.

25. ^ Maureen N. Ajuebor, Mark G. Swain. (2002). Role of chemokines and chemokine receptors in the gastrointestinal tract. Immunology, vol. 105 (2), 137-143. doi:10.1046/j.1365-2567.2002.01309.x.

26. a, b Hong Luo, Asok Chaudhuri, Valerie Zbrezezna, Yu He, A. Oscar Pogo. (2003). Deletion of Murine Duffy Gene (Dfy) Reveals that the Duffy Receptor Is Functionally Redundant. MCB, vol. 23 (8), 3030-3030. doi:10.1128/mcb.23.8.3030.2003.

27. ^ Tracey C. Dawson, Alex B. Lentsch, Zixuan Wang, John E. Cowhig, Antal Rot, Nobuyo Maeda. (2000). Exaggerated response to endotoxin in mice lacking the Duffy antigen/receptor for chemokines (DARC). doi:10.1182/blood.v96.5.1681.h8001681a_1681_1684.

28. ^ Tyler W. Benson, DanielS. Weintraub, Matthew Crowe, Nicole K.H. Yiew, Orishebawo Popoola, Ajay Pillai. (2018). Deletion of the Duffy antigen receptor for chemokines $(D A R C)$ promotes insulin resistance and adipose tissue inflammation during high fat feeding. Molecular and Cellular Endocrinology, vol. 473, 79-88. doi:10.1016/j.mce.2018.01.006.

29. ^ Carlos Rodriguez. (2020). The global helminth belt and Covid-19: the new eosinophilic link. Qeios. doi:10.32388/iwkqh9.2.

30. ^ François Devesa, Vida Chams, Premkumar Dinadayala, Alexandre Stella, Aude Ragas, Henri Auboiroux. (2002). Functional reconstitution of the HIV receptors CCR5 and CD4 in liposomes. doi:10.1046/j. 1432-1033.2002.03213.x.

31. ' Courtney Woolsey, Viktoriya Borisevich, Abhishek N. Prasad, Krystle N. Agans, Daniel J. Deer, Natalie S. Dobias. (2020). Establishment of an African green monkey model for COVID-19. doi:10.1101/2020.05.17.100289. 